\title{
Time of Arrival Analysis in NC DETECT to Find Clusters of Interest from Unclassified Patient Visit Records
}

\author{
Meichun Li*1, Wayne Loschen², Lana Deyneka ${ }^{3}$, Howard Burkom², Amy Ising ${ }^{1}$ and Anna \\ Waller $^{1}$
}

${ }^{1}$ Emergency Medicine, UNC Chapel Hill, Chapel Hill, NC, USA; 2Johns Hopkins University Applied Physics Laboratory, Laurel, MD, USA; ${ }^{3}$ North Carolina Division of Public Health, Raleigh, NC, USA

\section{Objective}

To describe a collaboration with the Johns Hopkins Applied Physics Laboratory (JHU APL), the North Carolina Division of Public Health (NC DPH), and the UNC Department of Emergency Medicine Carolina Center for Health Informatics (CCHI) to implement time-of-arrival analysis (TOA) for hospital emergency department (ED) data in NC DETECT to identify clusters of ED visits for which there is no pre-defined syndrome or sub-syndrome.

\section{Introduction}

TOA identifies clusters of patients arriving to a hospital ED within a short temporal interval. Past implementations have been restricted to records of patients with a specific type of complaint. The Florida Department of Health uses TOA at the county level for multiple subsyndromes (1). In 2011, NC DPH, CCHI and CDC collaborated to enhance and evaluate this capability for NC DETECT, using NC DETECT data in BioSense 1.0 (2). After this successful evaluation based on exposure complaints, discussions were held to determine the best approach to implement this new algorithm into the production environment for NC DETECT. NC DPH was particularly interested in determining if TOA could be used for identifying clusters of ED visits not filtered by any syndrome or sub-syndrome. In other words, can TOA detect a cluster of ED visits relating to a public health event, even if symptoms from that event are not characterized by a predefined syndrome grouping? Syndromes are continuously added to NC DETECT but a syndrome cannot be created for every potential event of public health concern. This TOA approach is the first attempt to address this issue in NC DETECT. The initial goal is to identify clusters of related ED visits whose keywords, signs and/or symptoms are NOT all expressed by a traditional syndrome, e.g. rash, gastrointestinal, and flu-like illnesses. The goal instead is to identify clusters resulting from specific events or exposures regardless of how patients present - event concepts that are too numerous to pre-classify.

\section{Methods}

In late 2011, NC DPH and JHU APL signed a Software License Agreement and soon thereafter CCHI received the TOA software package. In May 2012, the TOA controller was adapted and set up to run against ED visit data for all NC DETECT hospitals. The TOA looks for clusters in all ED visits by hospital based solely on arrival time in both 30 -minute and 60 -minute intervals. There is no pre-classification of the chief complaints or triage notes into syndromes. TOA alerts are viewable on the NC DETECT Web application and, as of August 2012, users are able to document any actions taken on these alerts.

\section{Results}

From April 15, 2012 to July 31, 2012, TOA generated 173 alerts across all 115 hospitals reporting to NC DETECT. The TOA identified a group of scabies-related ED visits that was not captured in another syndrome. The TOA also identified clusters identified by hospitals as disaster-related which included misspellings that had not been previously identified, e.g. "diaster" and "disater," as well as events involving out-of-town groups that will not be identified spatially (Table 1). This preliminary review of TOA alerts did not evaluate TOA for false negatives.

\section{Conclusions}

Our preliminary review of TOA shows that this algorithm approach can be helpful for identifying clusters of ED visits that are not captured by existing syndromes and can be used to identify hospital coding schemes for disaster events. The TOA will continue to be monitored in our production environment and evaluated for additional effectiveness. We will also explore tools that will display counts of terms within a TOA alert to assist in signal investigation.

Table 1: Sample clusters detected with TOA analysis

\begin{tabular}{|c|c|c|}
\hline Cluster Event Type & $\begin{array}{c}\text { Number of ED Visits in TOA alert / } \\
\text { Number of visits related to the specific } \\
\text { event cluster }\end{array}$ & Sample chief complaints in cluster \\
\hline Disaster-related & $13 / 9$ & $\begin{array}{c}\text { Disaster abd pain, diaster flu, disaster } \\
\text { phyc, diaster anxity, diaster black }\end{array}$ \\
\hline Scabies & $16 / 8$ & $\begin{array}{c}\text { Scabies 7FER, Scaobes 7FER, 5M } \\
\text { FOLLOW UP }\end{array}$ \\
\hline $\begin{array}{c}\text { Same out of town } \\
\text { location }\end{array}$ & $17 / 7$ & Cough, laceration(s) \\
\hline
\end{tabular}

\section{Keywords}

Cluster detection; Time-of-arrival analysis; Syndrome classification

\section{References}

1. Burkom H, Loschen W, Kite-Powell A et al. A Collaboration to Enhance Detection of Disease Outbreaks Clustered by Time of Patient Arrival, presented at the International Society for Disease Surveillance, 2010 Annual Conference, Park City, Utah, Dec 2, 2010

2. Deyneka L, Xu Z, Burkom H, Hicks P, Benoit S, Vaughan-Batten H, Ising A. Finding time-of-arrival clusters of exposure-related visits to emergency departments in contiguous hospital groups. Emerging Health Threats Journal 2011, 4: 11702 - DOI: 10.3402/ehtj.v4i0.11702

*Meichun Li

E-mail:mcli@email.unc.edu 